\title{
A roadmap to achieving readiness for macro adoption of distributed ledger technology (DLT) in the construction industry
}

\author{
Jennifer $\mathrm{Li}^{a} *$, Mohamad Kassem ${ }^{a}$ \\ ${ }^{a}$ Dept. of Mechanical \& Construction Engineering, Faculty of Engineering \& Environment, Northumbria University, Newcastle, NE1 8ST, UK
}

\begin{abstract}
Applications and uses cases of distributed ledger technology (DLT) are increasingly attracting interest in the construction industry. However, DLT in construction is still considered a nascent field of research and practical applications of DLT in construction are at the very early readiness stages. This paper builds on a previously developed socio-technical systems framework for DLT in construction (i.e. Li et al., 2019) built on four dimensions of technical, process, policy and social, and proposes a roadmap to achieving readiness for macro adoption of DLT in the construction industry. First, the paper reviews existing readiness and adoption models and technology roadmaps for new technological innovations in the context of DLT highlighting their strengths and detailing why they are not suitable for DLT. Then, drawing on experience of existing models as a basis, it proposes a four-stage roadmap to readiness for adoption of DLT in the construction industry. The four-stage DLT Roadmap incorporates Conceptualisation, Appraisal, Preparation and Implementation. This roadmap is intended to provide the industry with a comprehensive framework to support adoption and diffusion of DLT for specific use cases. Future work will involve proposal of guidelines for each of the four dimensions across the four-stage DLT Roadmap and testing through workshop-identified use cases of DLT in construction.
\end{abstract}

(C) 2019 The Authors. Published by Budapest University of Technology and Economics \& Diamond Congress Ltd.

Peer-review under responsibility of the scientific committee of the Creative Construction Conference 2019.

Keywords: distributed ledger technology (DLT); blockchain; technology roadmap; macro adoption; readiness; construction industry.

\section{Introduction}

Distributed ledger technology (DLT) will produce the most benefits when adoption reaches a critical mass and the community consists of many relevant members [1]; in the construction industry this necessitates extensive marketwide adoption of the system. This could involve mobile applications linked to DLT that record activities or performance as transactions on a distributed ledger as part of a construction project or facilities management of a building, or supply chain integration with Building Information Modelling (BIM) models where procurement decisions and shipping and delivery of goods and services are automatically uploaded to the ledger as the information model is simultaneously updated. These use cases bring about a number of challenges such as: (a) the consistency between the digital environment (e.g. information models, documentations, data), agreements (e.g. as in the information container), and the actual built assets (e.g. physical assets, goods, and services); (b) the need for recording key decisions about both the digital and the physical environment of a built asset across its lifecycle from conception through design, planning, construction, to operation and demolition [2,3]; and (c) the successful integration of required technologies BIM, DLT, Internet of Things (IoT) and smart contracts as discussed in [3]. The characteristics of DLT (e.g. redefining trust, decentralisation, anonymity) have the potential to support solutions to these challenges.

*Corresponding author: Jennifer Li, email: Jennifer.Li@ northumbria.ac.uk 
Li and Kassem / Proceedings of the Creative Construction Conference (2019) 001

https://doi.org/10.3311/CCC2019-001

This paper contributes to the body of research through proposal of a roadmap for achieving readiness for adoption of DLT in the construction industry. This a continuation of the extended socio-technical framework developed in [4] that assimilates four dimensions of technical, policy, process and social, and proposes two models - DLT FourDimensional Model and DLT Actors Model. A roadmap for new technologies is both a strategic and operational approach to support organisations and industries in charting technology issues relevant to their future success [5]. This is done by assessing current readiness models and roadmaps for new technology in construction and other industries, which were used as a basis for development of the DLT Roadmap for construction. The approach proposed in this paper may be applicable to other technological innovations and industries; however, terms and concepts may need to be redefined to be appropriate for the use/use case for which they are intended.

Several terms used throughout this paper do not have universally accepted definitions in the existing literature. Terms often have different meanings at different stages of a technology's adoption whether at the implementation stage or at the diffusion stage that follows [6]. For the purpose of this study, each of the terms' definitions are clarified: macro defines the scope of adoption which is at the industry scale within one defined market (e.g. a country) and includes the different user groups (individuals, organisational departments/teams, entire organisations, inter-organisational projects) such as those identified in the DLT Actors Model [4]. This study does not differentiate between these groups, it considers them as a whole making up the industry at the macro scale. Adoption is referred to as a 'state' or a 'milestone' that is achieved within a market once the corresponding construction sector perceives the use of DLT in construction as the norm. There will be varying levels of diffusion (i.e. rates of adoption) for the different use cases of DLT in construction by the time this milestone is reached. This milestone is preceded by 'implementation' which refers to the wilful act of inserting DLT into the construction industry for selected applications once readiness has been achieved for the corresponding use cases. Readiness is defined by the point at which the industry is in a suitable position to implement DLT across organisations and projects; it refers to the preparation and propension required to adopt DLT and involves being in a state where policies, processes and systems are sufficiently robust to withstand adoption of the new system.

The next section looks at technology adoption in construction and highlights existing models and roadmaps that supported this study; section 3 proposes the roadmap to achieving readiness for adoption of DLT in construction; and section 4 provides discussion and concludes the paper.

\section{Adoption of digital innovations}

Understanding how new technological innovations are adopted in construction generally will help in understanding the conditions required for DLT. This section considers the adoption of BIM to date along with the challenges it faced. Then, it highlights adoption/maturity models used to assess DLT and reviews existing models and roadmaps to support development of the DLT Roadmap proposed in this paper.

As BIM is the current expression of technological innovation in construction it is most appropriate to draw comparisons for DLT. BIM is a set of technologies, processes and policies affecting the industry's deliverables, relationships and roles [6]. While BIM offers many benefits and improvements to collaboration across the industry, there have been and still are many barriers to its widespread adoption. Barriers are offered including "lack of initiative and training, fragmented nature of the AEC industry, varied market readiness across geographies, and industry's reluctance to change existing work practice" ([7], p. 989). Key barriers include lack of senior management support, cost of implementation, scale of culture change required, other competing initiatives, lack of supply chain buy-in, staff resistance and ICT literacy, and legal uncertainties [8]. [9] add "the lack of tangible benefits for all parties involved or the understanding of the business value of BIM; the lack of experience within the workforce; the lack of universal use; the resistance to change; the contract type/project delivery method inhibiting technology adoption, and time and cost" (p. 9). While these are BIM-specific, each was identified as a possible barrier to adoption of DLT in construction and were central to developing a framework for implementation of DLT as seen in [4].

There is no academic literature on the adoption of DLT in the construction industry at the macro scale. Studies have been conducted across other industries and at different scales of analysis such as: logistics and supply chain management at an individual scale [10]; maturity of blockchain generally [11]; acceptance of Bitcoin and Blockchain at an individual scale [12]; and organisational adoption of blockchain [13]. 
Li and Kassem / Proceedings of the Creative Construction Conference (2019) 001

https://doi.org/10.3311/CCC2019-001

\subsection{Existing models for assessing readiness of innovations}

Models to assess readiness or maturity have been used for many years and many variations exist. They assess the gap between current and desired state in respect of an innovation or activity, however, they often omit roadmaps to achieving the desired state [14]. Roadmaps for adoption of new technology are more appropriate for DLT than maturity models at this stage in its development. Models and roadmaps key to this research are forthwith discussed.

Rogers' [15] Innovation-Decision Process supports evaluation and decisions of a new idea regarding adoption. The five stages are knowledge, persuasion, decision, implementation and confirmation. The process is offered as applicable at different scales including individual or other decision-making unit [15].

Technical Readiness Levels (TRL), the most widely used method of assessing an individual technology's maturity [1618], is effective in assessing the current level of a technology. However, it does not look at the surrounding nor operational environments of the technology; it does not offer guidance of the uncertainty to be expected during maturity of a system; nor does it offer a comparative analysis technique to assimilate against other TRLs [16]. The majority of complex systems fail at integration points with other technologies, therefore, [16] propose a readiness model to address these challenges, Systems Readiness Levels (SLR). SLR incorporates TRL and Integration Readiness Levels (IRL) measurement of the interactions of various technologies and comparison of the maturity between integration points [16]. The model is applicable to different applications and architectures [19] thus supports the socio-technical framework developed for implementation of DLT in construction. However, it focuses solely on technological systems and their interaction with other technologies; it is missing the interrelation between technologies and the remaining dimensions of process, policy and social as in the DLT Four-Dimensional Model. Similar readiness levels scales aligning to policy, process and social include a taxonomy of Policy Readiness Levels (PRL) [20] that mimics TRL [21] "for research directed towards the design of institutional and/or regulatory policies" ([20], p. 393); Process Readiness Levels (PRL) appearing alongside Material Readiness Levels (MRL) in the context of aerospace/space exploration [22,23]; and Societal Readiness Levels (SRL) to assess the level of societal adaptation of a social project, technology, product, process, intervention or social or technical innovation to be integrated into society [24].

Technology roadmaps follow six steps: 1) identify the needs and drivers; 2) identify products or services to meet the needs and the drivers; 3 ) identify technologies to support the products or services; 4) establish the linkages among the first three steps above; 5) develop plans to acquire or develop the technologies; 6) assign resources to accomplish the plans for acquisition and development [5]. A 12-step roadmap for organisational adoption of Information Technology and Infrastructure Library (ITIL) is proposed by [25] focusing on adoption within an organisation. [26] builds on the technology roadmap developed by [27] which is adaptable to both organisation and industry scales. Its three phases are: preliminary activity, development of the technology roadmap and follow-up activity. [27]'s process was developed with a focus on product/process driven needs; [26] extends the process for disruptive technologies. As DLT involves more than just adoption of a new technology [4], this process alone is not suitable to support its implementation. [28] use this process to develop a roadmap for smart city development in Korea. [29] proposes an Innovation Roadmap utilising a multi-layer, timeline-based structure incorporating technological change, industrial change, policy change and social change and the dynamics of regime and niche level developments within.

None of the models discussed in this section are suitable for the macro adoption of DLT in construction as a standalone tool to achieve readiness either because they were developed for a different organisational scale (i.e. individual, organisation), for a different industry, or simply do not fit the requirements for DLT. While some elements are applicable under the right circumstances, a number of the steps in existing models are elementary and carry little meaning. Construction is complex and requires changes across many faces of the industry before acceptance and adoption of new innovations and systems can be successful. This has been shown through the challenges faced by BIM, therefore, a specific roadmap that addresses the industry's complexity and track record of technological advancement is required. The next section proposes the DLT Roadmap that was influenced by some of the models discussed here.

\section{Proposed DLT Roadmap to achieve readiness for adoption of DLT in construction}

The proposed DLT Roadmap is a continuation of the socio-technical framework offered in [4]. As DLT is yet to be adopted in the construction industry to any noticeable extent, the purpose of this roadmap is to support the industry 
with its itinerary toward implementation and adoption. A roadmap at this stage will provide a more streamlined approach to adoption of a new system allowing the industry the opportunity to have all supporting infrastructure in place prior to implementation; it allows time to educate users/beneficiaries of the new system about its benefits whilst highlighting any potential challenges they should prepare for; and it supports the development of industry standards and regulations [30]. As discussed earlier in this paper, adoption of DLT in construction needs to happen at the macro scale at this early stage. DLT is a collaborative system that requires adoption throughout the supply chain and throughout the asset lifecycle; individual or organisational adoption alone will not be sufficient for its success as this does not increase trust between contracting parties nor does it promote intra-organisational decentralisation.

Technology roadmaps provide "a comprehensive approach for strategy planning to integrate science/technological considerations into product and business aspects as well as to provide a way to identify new opportunities in achieving a desired objective from the development of new technologies" ([5], p. 690). The DLT Roadmap (Fig. 1) is designed to support the construction industry in its decision to adopt DLT through offering a robust sequential process that will support development of a new set of processes and policies and aid in preparation and planning for its implementation. The roadmap is designed to support implementation of DLT for specific use cases rather than DLT generally recognising that some will require more preparation and legislation and will involve different actors and activities than others before successful adoption can occur. It consists of four stages: 1 Conceptualisation, 2 Appraisal, 3 Preparation and 4 Implementation. Conceptualisation provides understanding of what value DLT can add for a specific use case; appraisal considers the current environment and what needs to change to allow for adoption of DLT for the selected use case; preparation puts measures in place to ensure implementation of DLT is a success; and implementation sees those measures realised successfully. It considers the construction industry's current and desired state with respect to DLT and proposes progressive activities for the industry to follow at each stage that will guide informed decisions to continue toward adoption or whether to reject the new system between stages. These decision gates, indicated by the diamonds between stages in Fig. 1 offer the industry a structured process and introduce formal points where the benefits of continuing in terms of time, cost, opportunity costs, likelihood of success and appetite for the new system are reviewed. It is recommended that an industry appointed task force be established early (i.e. by government) to drive this roadmap as in [27]. The task force should consist of participation from diverse groups within the industry (i.e. industry practitioners, government, academics) who should be available for the duration of the roadmap giving the benefit of early buy-in from the people tasked with implementation [25].

\section{Conceptualisation}

Establish drivers, aims, objectives for adoption of DLT in construction. Identify how DLT will meet the needs of the industry for the use case. Review use of DLT for the same use case in other industries. Identify integration points of DLT with other technologies (i.e. BIM, IoT) and consider interoperability.

Identify the risks, benefits and challenges of DLT for the use case.

Determine critical success factors for the use case.

Identify resource requirements for use case.

Define the desired state following implementation of the use case.

\section{Preparation}

Identify whether sufficient level of resources are in place. Train workforce that will implement DLT.

Upgrade software, hardware, networks and infrastructure.

Reduce and mitigate risks, address challenges, exploit benefits.

Establish DLT standards, regulations, laws and compliance guidance. Develop workflows, processes and practices for new DLT system. Refine current and new systems through pilot studies and testing. Ensure the new DLT system meets critical success factors.

Finalise implementation plan.

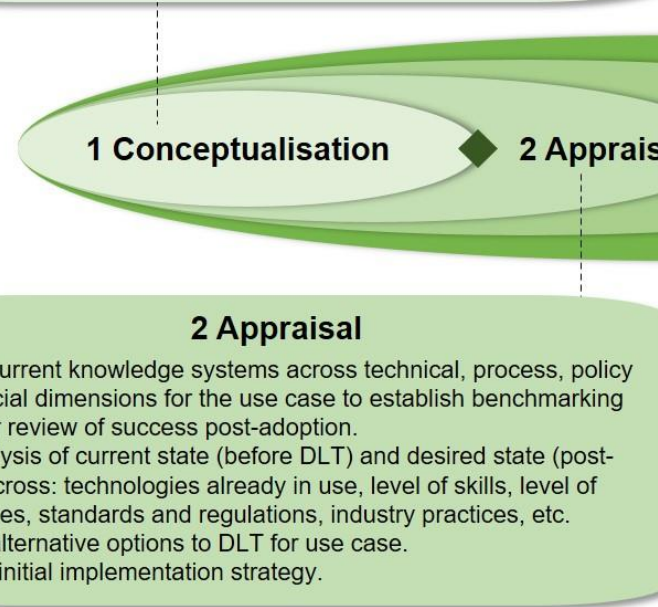

Fig. 1. DLT Roadmap 
Li and Kassem / Proceedings of the Creative Construction Conference (2019) 001

https://doi.org/10.3311/CCC2019-001

In further work, the four stages will be supported by a set of guiding components across the four dimensions (technical, process, policy, social) to for application to specific uses of DLT (i.e. automated supply chain payments, maintaining a historical digital record throughout the lifecycle of an asset). The four roadmap stages were devised from assimilating relevant aspects of existing models and roadmaps for adoption of new technologies as discussed in section 3 .

Fig. 1 provides details of the proposed activities that should take place during each stage that will be adapted for different use cases. The conceptualisation stage directs the task group to gain a comprehensive understanding of the use of DLT as part of the solution through research into the feasibility of DLT for the use case across the industry by completing the activities. This will enable the task force to make a decision on whether to continue towards adoption or reject DLT for this use case. The appraisal stage provides understanding of the current state of the industry for the use case by comparing it against the desired state with adoption of DLT. This enables the task force to identify changes that need to be enacted during the preparation stage before implementation can take place as well as providing benchmarking data against which to compare post-implementation data to measure success. The decision to continue towards adoption or to reject the use of DLT for the use case is made based on the appraisal activities where the decision to continue leads to the preparation stage. Here the task force should ensure that everything required for successful implementation, as identified through the conceptualisation and appraisal stages, is in place and that changes are made where necessary such as developing and/or updating software and hardware required to adopt DLT for the use case and to enact legislation so the new system operates within the confines of the law closing any potential loopholes for criminal and/or fraudulent activity, particularly where finances are involved. Following the final decision to adopt DLT for the use case in question, the implementation stage commences where the implementation plan is executed. During this stage, the benefits identified at the conceptualisation stage, along with others identified throughout the process, will begin to be realised. Success can be measured against critical success factors and benchmarking data. Challenges and benefits not perceived until implementation will be identified and mitigated or exploited as the new system is re-invented to ensure it is fit-for-purpose. From the implementation stage onwards, the system will be continuously refined and updated as DLT advances and as new use cases emerge.

\section{Discussion and conclusions}

This paper proposes a four-stage DLT Roadmap to support macro adoption of distributed ledger technology in the construction industry adaptable for specific use cases. It aims to assist development of a set of robust processes and policies to create an ecosystem that will support its implementation into a complex industry. The four-stages in the DLT Roadmap (conceptualisation, appraisal, preparation and implementation) were developed through considering contributions of other readiness models and technology roadmaps used in similar industries as no roadmap exists for DLT in construction. Each stage consists of a number of activities to be conducted that build on one another to create the ecosystem needed for successful implementation of the system. It is a developmental process that will support an appointed task force from conceptualisation through to implementation with structured adopt/reject points at the transition from one stage to the next and prescribes continuous reviews and updates of the implementation plan as the system advances and more is learned about how it will integrate and be implemented across the construction industry. The DLT Roadmap was developed specifically for the construction industry; its novelty lies in its ability to be adapted for any use case in the construction industry considering that the ecosystem required for successful adoption of DLT will differ from use cases to use case. For example, financial payments via smart contracts will require enactment of new financial legislation, task automation that does not include a financial payment will not. This will be demonstrated in future research as the DLT Roadmap is applied to specific use cases.

The DLT Roadmap forms part of the socio-technical framework for DLT that consists of the DLT Four-Dimensional Model and the DLT Actors Model to represent flexible, adaptable and scalable knowledge constructs and foundations that can be used for various investigations related to DLT applications in construction. DLT has the potential to support solutions to many of the challenges the construction industry faces with regards trust, collaboration and information sharing, regulation and compliance, payments and more [4] but it needs to be nurtured carefully to avoid challenges such as those experienced by the adoption of BIM.

This study's key limitation is that the DLT Roadmap was based on roadmaps developed for purposes other than DLT, some for individual or organisational application rather than industry-wide. There is the possibility that when the process is used in practice some elements will not meet the requirements needed for achieving readiness for adoption of the innovation. However, part of the roadmap is to review the process and update it based on real-world application. 
The next phase of the research will involve the proposal of guidelines for each of the four dimensions and their associated components across the four stages of the DLT Roadmap and be validated with industry practitioners and academics. It will consider integration with other technologies (i.e. BIM, IoT and smart contracts) as well as incorporating aspects such as creation of plans to train the required skills and educate the general public about DLT. In addition, a number of workshops will be held to identify a series of use cases with industry practitioners, academics and government to demonstrate how the DLT Roadmap and guidelines can be employed in practice.

\section{References}

[1] M. Dobrovnik, D. Herold, E. Fürst, S. Kummer, Blockchain for and in Logistics: What to Adopt and Where to Start, Logistics. 2 (2018) 18. https://www.doi.org/10.3390/logistics2030018.

[2] HM Government, Digital Built Britain: Level 3 Building Information Modelling - Strategic Plan, 2015. https://www.gov.uk/government/uploads/system/uploads/attachment_data/file/410096/bis-15-155-digital-built-britain-level-3-strategy.pdf.

[3] J. Li, M. Kassem, A.L.C. Ciribini, M. Bolpagni, A proposed approach integrating DLT, BIM, IOT and smart contracts: Demonstration using a simulated installation task, in: Int. Conf. Smart Infrastruct. Constr., Cambridge, 2019.

[4] J. Li, D. Greenwood, M. Kassem, Blockchain in the built environment and construction industry: A systematic review, conceptual models and practical use cases, Autom. Constr. 102 (2019) 288-307. https://doi.org/10.1016/j.autcon.2019.02.005.

[5] T.U. Daim, T. Oliver, Implementing technology roadmap process in the energy services sector: A case study of a government agency, Technol. Forecast. Soc. Change. 75 (2008) 687-720. https://doi.org/10.1016/j.techfore.2007.04.006.

[6] B. Succar, M. Kassem, Macro-BIM adoption: Conceptual structures, Autom. Constr. 57 (2015) 64-79. https://doi.org/10.1016/ j.autcon.2015.04.018

[7] N. Gu, K. London, Understanding and facilitating BIM adoption in the AEC industry, Autom. Constr. 19 (2010) $988-$ 999. https://doi.org/10.1016/j.autcon.2010.09.002.

[8] R. Eadie, H. Odeyinka, M. Browne, C. McKeown, M. Yohanis, Building Information Modelling Adoption: An Analysis of the Barriers to Implementation, J. Eng. Archit. 2 (2014) 77-101. https://doi.org/10.1007/s13398-014-0173-7.2.

[9] M. Kassem, T. Brogden, N. Dawood, BIM and 4D planning: a holistic study of the barriers and drivers to widespread adoption, KICEM J. Constr. Eng. Proj. Manag. 2 (2012) 1-10. https://doi.org/10.6106/JCEPM.2012.2.4.001.

[10] M.M. Queiroz, S. Fosso Wamba, Blockchain adoption challenges in supply chain: An empirical investigation of the main drivers in India and the USA, Int. J. Inf. Manage. 46 (2019) 70-82. https://doi.org/10.1016/j.ijinfomgt.2018.11.021.

[11] H. Wang, K. Chen, D. Xu, A maturity model for blockchain adoption, Financ. Innov. 2 (2016) 12. https://doi.org/10.1186/ s40854-016-0031-z.

[12] D. Folkinshteyn, M. Lennon, Braving Bitcoin: A technology acceptance model (TAM) analysis, J. Inf. Technol. Case Appl. Res. 18 (2016) 220-249. https://doi.org/10.1080/15228053.2016.1275242

[13] F. Holotiuk, J. Moormann, Organizational Adoption of Digital Innovation : the Case of Blockchain Technology, Twenty-Sixth Eur. Conf. Inf. Syst. (2018). https://aisel.aisnet.org/ecis2018_rp/202.

[14] T. Mettler, P. Rohner, Situational maturity models as instrumental artifacts for organizational design, in: Proc. 4th Int. Conf. Des. Sci. Res. Inf. Syst. Technol. - DESRIST '09, ACM Press, New York, New York, USA, 2009: p. 1. https://doi.org/10.1145/1555619.1555649.

[15] E. Rogers, Diffusion of Innovations, 5th ed., Free Press, New York, NY, 2003. https://doi.org/10.1287/opre.51.6.866.24918.

[16] B. Sauser, D. Verma, J. Ramirez-Marquez, R. Gove, From TRL to SRL: The concept of systems readiness levels, Conf. Syst. Eng. Res. Los Angeles, CA. (2006) 1-10. http://www.boardmansauser.com/downloads/2005SauserRamirezVermaGoveCSER.pdf.

[17] J.C. Mankins, Technology readiness assessments: A retrospective, Acta Astronaut. 65 (2009) $1216-1223$. https://doi.org/10.1016/J.ACTAASTRO.2009.03.058.

[18] A. Olechowski, S.D. Eppinger, N. Joglekar, Technology readiness levels at 40: A study of state-of-the-art use, challenges, and opportunities, in: 2015 Portl. Int. Conf. Manag. Eng. Technol., IEEE, Portland, OR, 2015: pp. 2084-2094. https://doi.org/10.1109/PICMET.2015.7273196.

[19] B.J. Sauser, J.E. Ramirez-Marquez, D. Nowicki, A. Deshmukh, M. Sarfaraz, Development of Systems Engineering Maturity Models and Management Tools: Final Technical Report 2011-TR-014, Hoboken, NJ, 2011. http://www.dtic.mil/dtic/tr/fulltext/u2/a546788.pdf.

[20] L. Tesfatsion, Modeling economic systems as locally-constructive sequential games, J. Econ. Methodol. 24 (2017) $384-409$. https://doi.org/10.1080/1350178X.2017.1382068.

[21] L. Tesfatsion, Electric power markets in transition: Agent-based modeling tools for transactive energy support, Elsevier B.V., 2018. https://doi.org/10.1016/bs.hescom.2018.02.004.

[22] S. James, Nondestructive Testing and Material/Process Readiness Levels, Tech Spotlight. https://www.asminternational.org/documents/10192/1756177/amp16010p093.pdf/ad1868e7-bccd-474d-b36b-4da26a558055. (2002) 93.

[23] B.N. Bhat, Materials, Processes and Manufacturing in Ares I - Upper Stage: Integration with Systems Design and Development, Huntsville, AL, United States, 2008. https://ntrs.nasa.gov/archive/nasa/casi.ntrs.nasa.gov/20080037300.pdf.

[24] Innovation Fund Denmark, Societal Readiness Levels (SRL) defined according to Innovation Fund, (n.d.). https://innovationsfonden.dk/sites/default/files/societal_readiness_levels_-_srl.pdf (accessed 26 June 2018).

[25] N. Ahmad, N.T. Amer, F. Qutaifan, A. Alhilali, Technology adoption model and a road map to successful implementation of ITIL, J. Enterp. Inf. Manag. 26 (2013) 553-576. https://doi.org/10.1108/JEIM-07-2013-0041.

[26] S.T. Walsh, Roadmapping a disruptive technology: A case study The emerging microsystems and top-down nanosystems industry, Technol. Forecast. Soc. Change. 71 (2004) 161-185. https://doi.org/10.1016/j.techfore.2003.10.003.

[27] M.L. Garcia, O.H. Bray, Fundamentals of technology roadmapping, Albuquerque, NM, and Livermore, CA (United States), 1997. https://doi.org/10.2172/471364.

[28] J.H. Lee, R. Phaal, S.H. Lee, An integrated service-device-technology roadmap for smart city development, Technol. Forecast. Soc. Change. 80 (2013) 286-306. https://doi.org/10.1016/j.techfore.2012.09.020.

[29] T. Könnölä, Innovation Roadmap: Exploring Alternative Futures of Industrial Renewal, 2007. http://iri.jrc.ec.europa.eu/documents/10180/12569/Innovation roadmap - Exploring alternative futures of industrial renewal (accessed 8 February 2019).

[30] R.J. Kauffman, D. Ma, M. Yu, A metrics suite of cloud computing adoption readiness, Electron. Mark. 28 (2018) 11-37. https://doi.org/10.1007/s12525-0213-y 